\title{
A Two Level Face Recognition Algorithm For Efficient Attendance Marking
}

\author{
Bhaskar $\mathrm{B}^{1}$,Shwetha $\mathrm{B}^{2}$, Rashmi K ${ }^{3}$, Dr.K.V.Mahendra Prashanth ${ }^{4}$ \\ ${ }^{1}$ Associate Professor, \\ ${ }^{2}$ Postgraduate Student, \\ ${ }^{3}$ Postgraduate Student \\ ${ }^{4}$ Professor \\ ${ }^{1}$ bhaskar.brv@gmail.com, ${ }^{2}$ Shwethabelliappa23@gmail.com, ${ }^{3}$ rashmik2121@gmail.com \\ Department of Electronics and communication S.J.B Institute of Technology, Bengaluru-60
}

\begin{abstract}
A face recognition is ascending as an exuberant research region traversing many controls like image processing, design acknowledgment, pattern recognition, PC vision and neural systems. Face recognition innovation has different business applications and another business. Here this paper introduces an automated complex face recognition based on the combination of $w$-Quartette and $v$-HOG which prepares the face picture for recognition and interpret the extraction information. As a piece of the proposed calculations, Filters of Wavelets have been executed utilizing multi-resolution approaches because of their adaptability, reasonableness, and normality for face acknowledgment. The proposed framework enhances the execution of a current face recognition framework by reducing out the occlusions, human intervention, marking and entry of attendance.
\end{abstract}

Index Terms: Biometrics, Wavelet transform, w-Quartette, v-HOG

\section{Introduction}

Biometric makes use of the physiological or behavioral characteristics to identify and substantiate the person. In order to overcome human intervention, the biometrics have been automated. There is uniqueness in the characteristics of the physiological and behavioral measurements. Finger based scan, hand based scan, iris based scan, retina based scan and facial based scan.

A face isn't just an accumulation of face expression rather it is an intentional thing. Face recognition could be a typical investigation theme with the organizing of uses in numerous modern regions together with police work and security, virtual relativity and entertainment and human-machine communications. Customarily the teachers place the attendance of students, and they ought to guarantee the accuracy of the check attendance of the individual student. Accordingly, the address term gets squandered and furthermore, the intermediary participation can't be kept away from with this traditional way of taking attendance.

There are huge number of techniques which have been applied for the face recognition. The faces where treated as points in the space of high dimension and there calculated the distance of Euclidean between them during former days. The dimensional reduction techniques are added to the problem such as Principle Component Analysis (PCA) in order to reduce the difficulty of the recognition techniques. A further [6] Discrete wavelet transform technique here used to perform the feature extraction. The wavelets have been decomposed into divisions and have been implemented to test the best performances among them. This paper defines how the face images can be represented in terms of eigenfaces, which facilitates gradual changes in face and also for easy computation. These face images are mapped onto w-Quartette color space to effectively interpret data existing in the picture. Further, the variant Hoglets are employed for the feature extraction which extracts the discriminative features displaying linear properties to map line singularities and at a same time to derive the features from the tender features the face. The extracted features are then projected to a lower dimensional space for the efficient face recognition. Five distinctive measures are considered to obtain an average correctness rate.

\section{Focus area}

Since biometrics can be implemented by companies, governments, customs, churches hospitals banks and military (police, army, navy, air force) to verify the identity of persons, here its use in the academic , Knowledge intensive area (education, industries), commercial, production are explained.

\section{Literature survey}

Olufemi Sunday Adeoye [1], here explores the varied strategies of biometric identification that has evolved over the years and also the features used for every modality. Different types of emerged and emerging technologies related to biometrics are briefly explained. W. Zhao, R. Chellappa, P. J. Phillips a. Rosenfeld [2], 
this paper explained the latest survey on the face recognition technological researches. It had given the updated review on the research. Bhaskar. Belavadi, K. Mahanthesh and G.P Geetha [3], this paper gives the explanations about how fSVD and Ridget transform reduces the illumination and normalizes the image. Here they also explain how the fSVD normalizes the face image by reducing the illumination by modeling the SVD. Dr. Pankaj Tomar, Preeti Mehta [5], this paper explains face recognition and automated attendance management using the raspberry Pi camera module and Matlab. The camera sections are operated with the help of raspberry $\mathrm{Pi}$ and the face recognition section is done using the coding in matlab.

Images suffer from the illumination and pose variations Gheorghita Ghinea, Rajkumar Kannan, And Suresh Kannaiyan [8] proposed a system which showed invariant to illumination and pose variationswith the help of image gradients orientation. Jianmei Lei, Qingwen Han, Lei Chen, Zhida Lai, Lingqiu Zeng, And Xin Liu[9] here another color space displaying multi-threshold decision criteria used to enhance facial feature extraction and a methodology is used to remove and reduce the noise and other impacts. Tests demonstrate in the system that the side face contour lines can be successfully extracted using the proposed algorithm.

\section{A. Block diagram}

\section{Proposed Methodology}

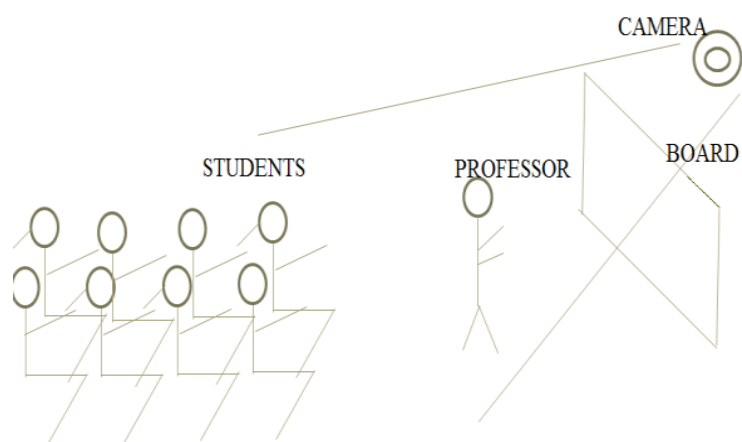

Fig 1. Classroom scenario in the automated face recognition technology.

Above indicated Figure 1 clarifies the classroom situation. An entire framework begins with educator entering the classroom. Each of the classrooms is given distinctive ID. As soon Professor enters the classroom the camera begins taking the Pictures consecutively, begins handling the information. Understudies are made to sit in the class confronting the board or Professor since framework takes after the up close and personal classroom framework. The cameras utilized here can be a Bluetooth camera, CCTV, Mobile camera or some other face catching gadgets.

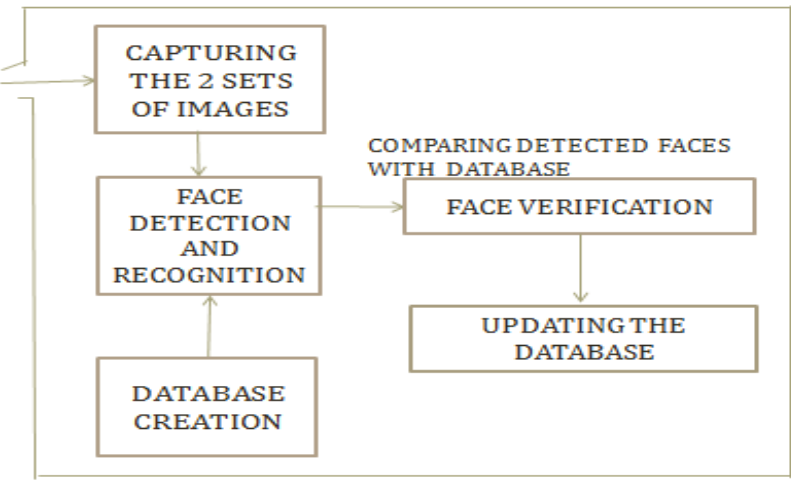

Fig 2. Image processing and face recognition.

Fig 2 explains further processing after the capturing the images.

\section{B. Flow Chart}

The Fig 3 explains the flow chart of the complete proposed system and the next block explains the process followed in the image processing of the proposed system. 

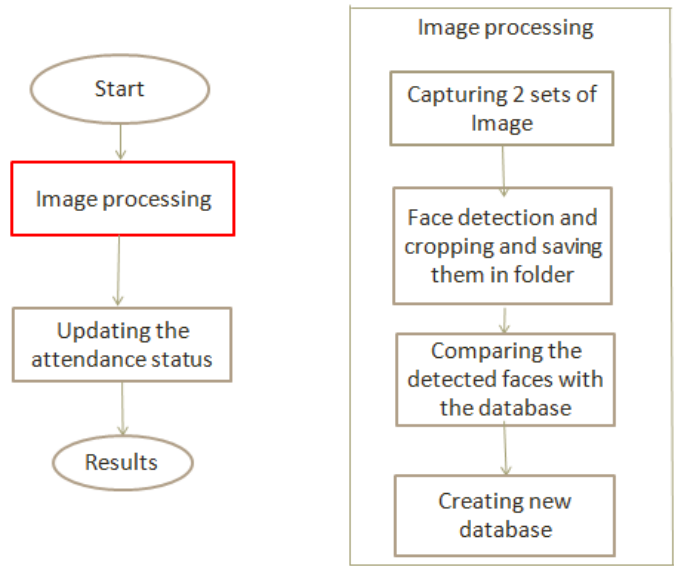

Fig 3. Flow chart of the proposed system and the block diagram of image processing

\section{Algorithm for database creation and recognition}

In this section the proposed face image detection and classification technique are discussed. Taking out the primary accuracy reduction techniques dealing with reduction of the effects ,First the face images are mapped onto w-Quartette color space to effectively interpret information existing in the image. The variant Hoglets extracts the discriminative features exhibiting the linear properties to map line singularities and at the same instance they derive the features of face contortion. In order to foster the extracted features the extracted features are projected to a lower dimensional space for the efficient face recognition. To obtain the average correctness rate five different classification similarity measures are used.

\section{i. w-Quartette Colorspace}

All the color models have different discriminative index. The color images will have more information compared to the grey scale images which helps to improve the face recognition. Using such properties deriving an image from the original image by mapping it on Quartette Colorspace.

Taking the colored face image as $\mathrm{F}(\mathrm{p}, \mathrm{q}, \mathrm{r})$ of size $(a * b * 3)$ is mapped as follows,

$\mathrm{F}_{\mathrm{ci}}(\mathrm{p}, \mathrm{q}, \mathrm{r})=\{\mathrm{F}(\mathrm{p}, \mathrm{q}, \mathrm{r}) \rightarrow\{\mathrm{w} * \mathrm{Lab}, \mathrm{x} * \mathrm{LUV}, \mathrm{y} * \mathrm{YCgCr}, \mathrm{z} * \mathrm{YUV}\}\}$

The face image F(p,q,r) is mapped to four colorspaces Lab, LUV, YCgCr, YUV , where $\mathrm{i}=1,2,3,4$, and $\mathrm{w}, \mathrm{x}, \mathrm{y}, \mathrm{z}$ are the weights for Colorspace, they are set based on the behavior of the colorspace with fSVD.

$$
I_{f i}(p, q, r)=\frac{\left(F_{c i}(p, q, r)+\beta_{1} * H_{i}(p, q, r)\right)}{\left(1+\left(\beta_{1}, \beta_{2}, \beta_{3}, \beta_{4}\right)\right)}
$$

where $\mathrm{H}_{\mathrm{i}}(\mathrm{p}, \mathrm{q}, \mathrm{r})=\mathrm{U} * \sum^{\gamma_{\mathrm{i}}} * \mathrm{~V}^{\mathrm{T}}$, Flustered SVD(fSVD) is applied in order to reduce the illumination and to normalize the image.

$\left[U, \sum, V\right]=$ SingularValuedecomp $(\operatorname{Inorm}(\mathrm{p}, \mathrm{q})) \quad$ where

$$
\beta_{1}=0.07, \beta_{2}=0.05, \beta_{3}=0.05, \quad \beta_{4}=0.05, \gamma_{1}=0.695, \gamma_{2,3,4}=0.895
$$

\section{ii. $\quad$ Variant Hoglets}

Histogram of Oriented Gradient features described by Navneed and Dalal and Bill triges [6], this signifiers helps in signifying the object appearance and shape within an image can be explained by the distribution of local intensity gradients or adjoin directions. More effective output is obtained if the image is subjected to the division and the small colligated regions called cells, When histogram of gradient is applied to the each cell it starts gathering the gradient directions or adjoin orientations for the pixels within the cell. thus the combination of these histograms them represents the signifiers. the advantages of HOG signifiers helps in the better face recognition technique[7]. In order to improve the accuracy rate of face recognition, variant Hoglets make use of various gradient filters adopted based on color space. In combining the behavior of wavelets with HOGs under different colorspace, the incubated daubechies filter kernels for HOG given as , 
$\mathrm{dbn}=$ coeff 1, coeff 2 coeffn,

where $\mathrm{n}=7$ and 9 are used in combination with $\mathrm{YCgCr}$ and Lab colorspace.

\section{iii. Singular value decomposition (SVD)}

There is a bulk matrix of grayscale values in a picture, Each pixel, color is depicted by a matrix for every of them. The image possesses the tendency of obtaining compressed whenever close pixels are correlative (not random). Matrix A is separated by SVD into rank one pieces uv ${ }^{\mathrm{T}}=$ (column)(row). Eigenvectors are the columns and rows here that is of symmetric matrices $\mathrm{AA}^{\mathrm{T}}$ and $\mathrm{A}^{\mathrm{T}}$. Typical definition of $\mathrm{SVD}$ is given as,

Applying the linear algebra of "Singular value Decomposition (SVD)" , is the main goal of the project here, to midlevel image process, particularly to the area of image compression, detection and recognition. The 3 new matrices $U, S, V$ are products after the factorization of matrix $A$, in such approach that $A=U S V^{\mathrm{T}}$. $U, V$ are considered as orthogonal matrices and square matrix here is $\mathrm{S}$.

\section{iv. Distance measures}

Features obtained from the above proposed is compressed by projecting them on to the lower dimensional subspace and five different classification measures are used to get the average correctness rate.

\section{Implementation}

Following steps will explain the implementation section:

i) Student's database creation

ii) Face detection and Face recognition with w-Quartette and v-HOG

iii) Updating the database

\section{i) Student's database creation}

Complete database of students is created, in the proposed system the database is initially created for 9 students. Data base will consist the class room's ID, student's ID, Professor's ID. below Fig 4 is the database created.
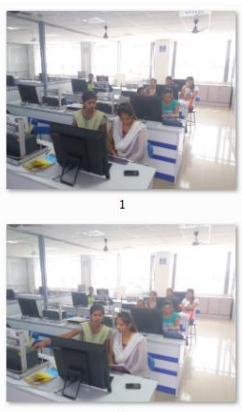

Fig 4: Class room images taken from camera
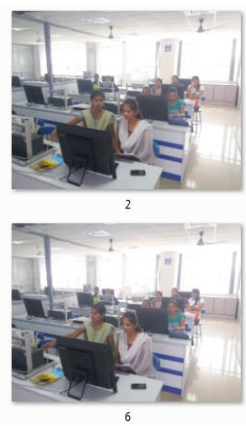
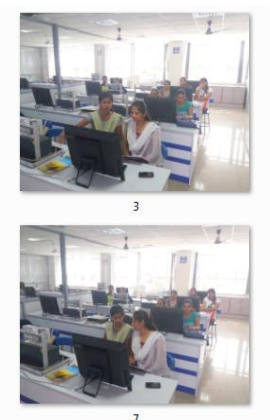

\section{ii) Face detection and Face recognition}

Face detection: skin color based Face detection method is used in proposed system. A frame of classroom image is captured with a gap of few minutes. The results for application of the face detection approach is as shown in the Fig 5 and Fig 6.

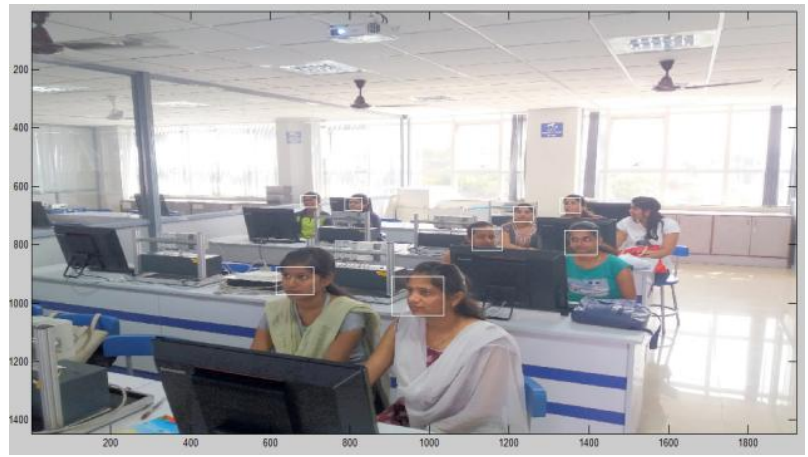

Fig 5: Face detection in a frame with boundary box. 


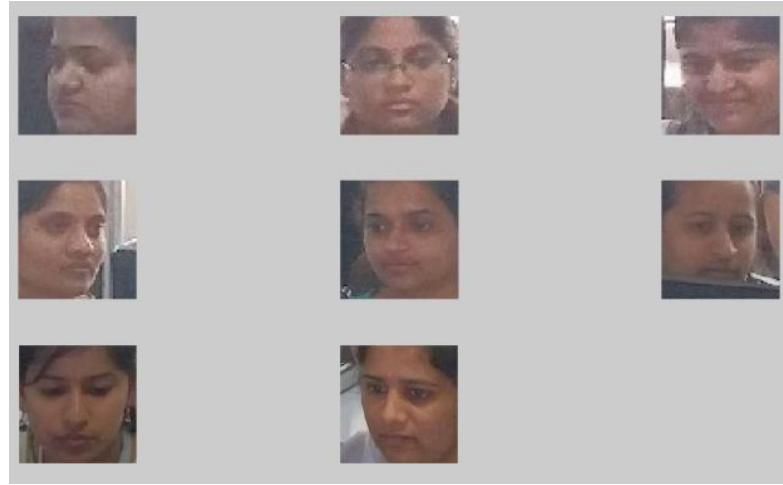

Fig 6: Cropped faces from a single frame

Face recognition: The face recognition performed by mapping the face picture onto w-Quartette color, which prepares the picture for the face acknowledgment in an ideal way. fSVD then applied to normalize the mapped picture. Further to extricate the shape descriptors $\mathrm{v}-\mathrm{HOG}$ is connected to the derived confront picture and they are projected on to the low dimensionality eigen subspace.

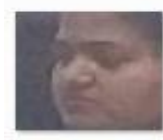

$1 \_12$

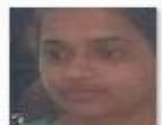

4_12

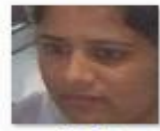

7_12

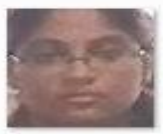

2.12

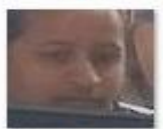

5_12

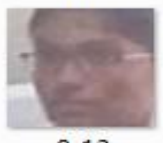

8_12

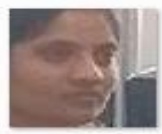

3_12

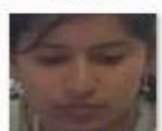

6_12

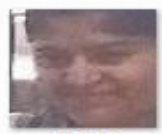

9_12

Fig 7: final database

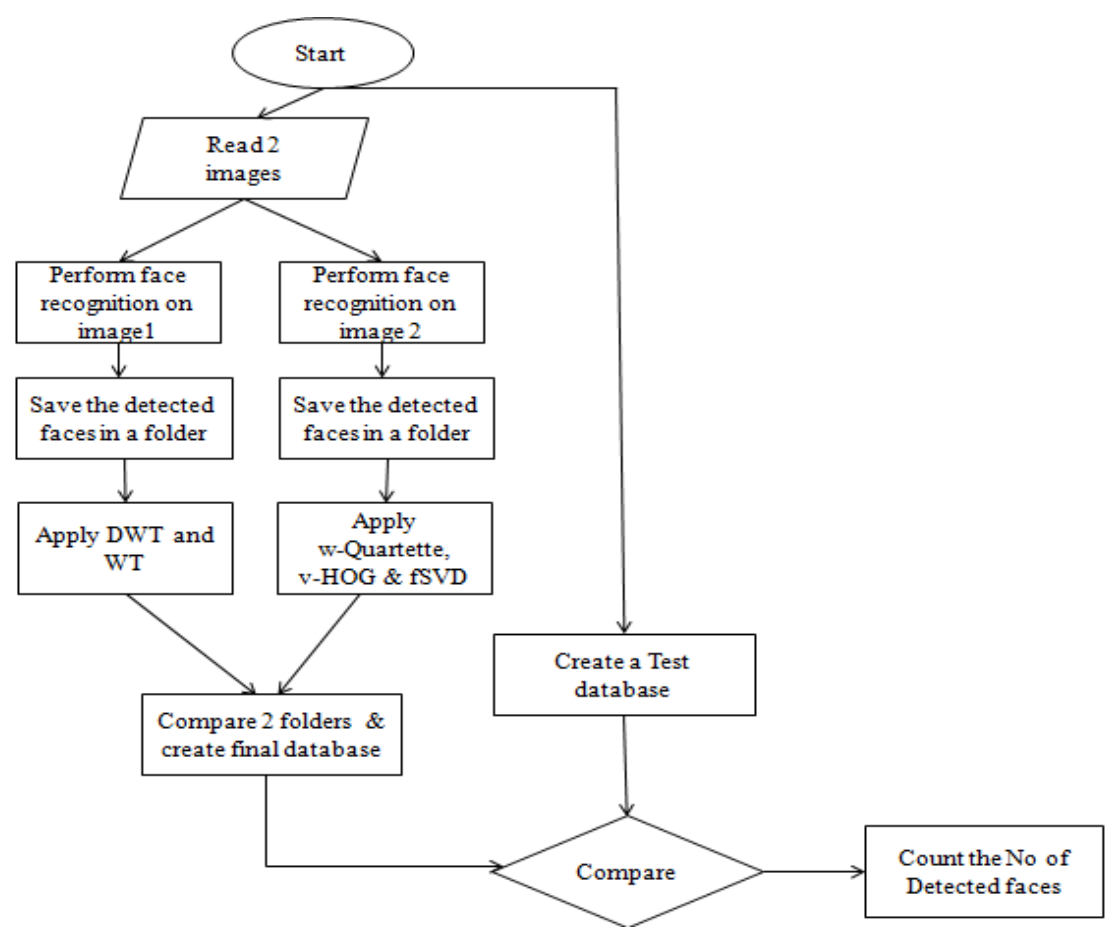

Fig 8 : Flow Chart of proposed system. 


\section{iii) Updating the database and marking attendance}

The HOG signifiers have got few advantages over the geometric and photometric transformation. Making use of these advantages which in turn helps in the face recognition accuracy. The results obtained from the v-HOG signifiers thus improves the proposed system, updating of the database took care of.

Figure 7 explains the final database structure and figure 8 explains us the complete flow chart of the proposed system .

\section{Conclusions}

This project has developed an efficient algorithm as a productive calculation for a complex face recognition with combination of w-Quartette Color space with Variant Hoglets (V-HOG) and in coactions fSVD. WQuartette color space has the ability to interpret suppressed information of images. Variant Hoglets gives substantive elements displaying linear properties to guide line singularities and also helps in extracting the features of face contortions. furthermore extricates the elements of face distortions. The recreation comes about recommends the proposed method yields the comes about under single per individual. The final outcome will be all in all a single package product which can work as a standalone attendance management system without any human intervention the process of managing and attendance.

\section{References}

[1] Olufemi Sunday Adeoye,"A Survey of Emerging Biometric Technologies", International Journal of Computer Applications (0975 8887) Volume 9- No.10, November 2010.

[2] W. Zhao,R. Chellappa, P. J. Phillips a. Rosenfeld" Face recognition A literature survey", ACM Computing Surveys, Vol. 35, No. 4, December 2003, pp. 399-458.

[3] Bhaskar. Belavadi, K. Mahanthesh ang G.P Geetha,"An Investigation of fSVD and Ridgelet Transform for llumination and Expression Invariant Face Recognition", Advances in Intelligent Informatics. Springer International Publishing. (2015), 31-38.

[4] Samuel Lukas, Aditya Rama Mitra, Ririn Ikana Desanti, Dion Krisnadi ,"Student Attendance System In Classroom Using Face Recognition Technique" , IEEE Conference Publications, 2016 International Conference on Information and Communication Technology Convergence (ICTC), Page 1032 - 1035

[5] Dr. Pankaj Tomar,Preeti Mehta, "An Efficient Attendance Management Sytem based on Face Recognition using Matlab and Raspberry Pi 2", IJETSRISSN 2394 - 3386,Volume 3, Issue 5,May 2016

[6] Dalal, Navneet, and Bill Triggs . "Histograms of oriented Gradients for human detection."In Computer Vision and Pattern Recognition, 2005. CVPR 2005, IEEE Computer society Conferance on, vol, 1(2005).

[7] Tzimiropoulous.G, Zafeirio. S, Pantic.M, "Principal component analysis of image gradient orientations for face recognition. Automativ Face Gesture Recignition and workshops, 2011 IEEE Conferance.

[8] Gheorghita Ghinea, Rajkumar Kannan, And Suresh Kannaiyan, "Gradient-Orientation-Based PCA Subspace for Novel Face Recognition".IEEE access the journel for open access publishing. Digital Object Identifier 10.1109/ACCESS.2014.2348018

[9] Jianmei Lei, Qingwen Han, Lei Chen, Zhida Lai, Lingqiu Zeng, And Xin Liu, "A Novel Side Face Contour Extraction Algorithm for Driving Fatigue Statue Recognition". IEEE access the journel for open access publishing.Digital Object Identifier 10.1109/ACCESS.2017.2686424.

[10] M. Hassan, I. Osman, and M. Yahia, "Walsh-Hadamard Transform for Facial Feature Extraction in Face Recognition", World Academy of Science, Engineering and Technology International Journal of Computer, Electrical, Automation, Control and Information Engineering Vol:1, No:5, 2007.

[11] Lijie Cao, "Singular Value Decomposition Applied To Digital Image Processing", Division of Computing Studies Arizona State University Polytechnic Campus Mesa, Arizona 85212.

[12] Pallavi D.Wadkar, Megha Wankhade, "Face Recognition Using Discrete Wavelet Transforms", International Journal of Advanced Engineering Technology E-ISSN 0976-3945.

[13] Gautham Sitaram Yajia, Sankhadeep Sarkara, K Manikantana, S Ramachandran b "DWT feature extraction based face recognition using intensity mapped unsharp masking and laplacian of gaussian filtering with scalar multiplier", Published by Elsevier Ltd. Procedia Technology 6 ( 2012 ) $475-484$.

[14] Nazare Kanchan Jayant, Surekha Borra , " Attendance Management System Using Hybrid Face Recognition Techniques", 2016 Conference on Advances in Signal Processing (CASP) Cummins College of Engineering for Women, Pune. Jun 9-11, 2016. 